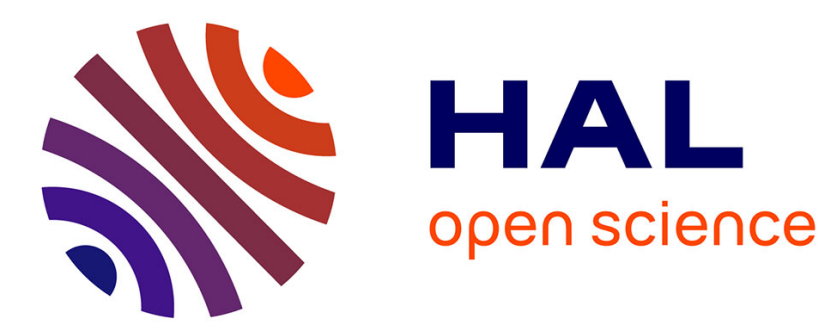

\title{
The Kolmogorov-Taylor Law of turbulence: what can rigorously be proved?
}

\author{
Roger Lewandowski
}

\section{To cite this version:}

Roger Lewandowski. The Kolmogorov-Taylor Law of turbulence: what can rigorously be proved?.

Handbook of Applications of Chaos Theory, 2016. hal-02331035

\section{HAL Id: hal-02331035 \\ https://hal.science/hal-02331035}

Submitted on 24 Oct 2019

HAL is a multi-disciplinary open access archive for the deposit and dissemination of scientific research documents, whether they are published or not. The documents may come from teaching and research institutions in France or abroad, or from public or private research centers.
L'archive ouverte pluridisciplinaire HAL, est destinée au dépôt et à la diffusion de documents scientifiques de niveau recherche, publiés ou non, émanant des établissements d'enseignement et de recherche français ou étrangers, des laboratoires publics ou privés. 


\title{
The Kolmogorov-Taylor Law of turbulence: what can rigorously be proved?
}

\author{
Roger Lewandowski *,
}

\begin{abstract}
We define a mathematical framework in which we can specify the Reynolds decomposition and the correlation tensors of an incompressible locally homogeneous and isotropic turbulent flow. After having fixed the technical background and some probabilistic tools, we focus on the 2-order correlation tensor, which is the covariance matrix of the velocity vectors at two different points of the flow. We perform a Taylor expansion of this matrix when the two points are close to one another. We characterize the principal part of this expansion, for which we prove the law of the $2 / 3$ by a mathematical similarity principle.
\end{abstract}

MCS Classification : 35Q30, 76D05, 76D06, 76F05

\section{Introduction}

Most of realistic flows are turbulent, looking chaotic, disordered, unpredictable. The physical processes that govern turbulence are far to be all understood, although it attracted a lot of attention in the literature since the $19^{\text {th }}$ century. Therefore, turbulence remains one of the main challenge of modern science, which has an impact from the human, social and economic standpoints, especially in the understanding of climate change and ecological issues.

Based on works carried out by Boussinesq [1] and Reynolds [27], it has been soon recognized that the velocity field of a turbulent flow can be decomposed as the sum of a mean field and a fluctuation. Initially, the mean velocity field was specified by its long time averaged. Later, Taylor [29] introduced the notion of statistical means, where the velocity and the pressure of the flow are considered as abstract random fields. The mean velocity is then its mathematical expectation, which also allowed Taylor to define the notion of isotropic turbulence, through algebraic properties satisfied by the correlation tensors.

In [21], Kolmogorov mainly revisited Taylor's work. He generalized the notion of isotropy by introducing local isotropy, stating somehow that all turbulent flows are locally isotropic. In this context, he retrieved the law of the $2 / 3$, already found in [29]. However, the main impact of Kolmogorov's paper lies in a footnote, in which he explains that turbulence can be depicted by a structure of eddies of different scales that interact with each other, the scales being distributed in a continuous range. The large scale eddies transfer energy to smaller ones, that transfer energy to more smaller, and so on until a final scale that dissipates energy as heat.

Kolmogorov's paper is written from a physical viewpoint, without considering the NSE. The aim of the present work is to define a mathematical framework in which notions

*IRMAR, UMR 6625, University of Rennes 1, Campus Beaulieu, 35042 Rennes cedex FRANCE; Roger.Lewandowski@univ-rennes1.fr, http://perso.univ-rennes1.fr/roger.lewandowski/ 
such as homogeneity and isotropy can be set up, the correlation tensors of the flow can be properly defined, and in which we can find the right mathematical hypotheses that allow to rigorously describe the structure of the covariance matrix in order to prove standard laws of the turbulence, such as the law of the $2 / 3$. To do so, we are led to make connections between the physical concepts of $[21,29]$ and modern mathematical results about the Navier-Stokes equations (NSE).

We start with a short overview about the Navier-Stokes equations, considering throughout this text the incompressible case. Then, we define the length-time bases that provide a framework for performing the dimensional analysis, which yields to introduce the notion of generalized Reynolds number and to give the dimensionless form of the NSE. We state the Reynolds similarity principle in this context and seek for solutions of the NSE that verify this similarity principle. We next define general homogeneous and isotropic tensors. We prove algebraic results in order to fully characterize isotropic tensors of orders 1 and 2 .

Once we are done with this technical background, we recall the suitable probabilistic framework, which was initially introduced in [5] by considering smooth solutions of the NSE. The underlying probabilistic space is a set of initial data, over which we are able to construct a probability measure. This construction should be compared with the notion of statistical solutions, developped for instance in [16].

The correlation tensors can therefore be specified, yielding the definition of homogeneous and istropic turbulence. We also introduce by this way the Reynolds stress, that plays a central role in the turbulence models.

We then consider the correlation tensor of order 2 in a ball of fluid $\delta V$, centered at a point $\mathbf{x}_{0}$, and over a short time interval. From the probability viewpoint, this tensor, denoted here by $B\left(\mathbf{x}, \mathbf{x}_{0}\right)$, is the covariant matrix of the velocities at $\mathbf{x}_{0}$ and any other point $\mathbf{x}$ of the ball of fluid. Therefore, $B\left(\mathbf{x}, \mathbf{x}_{0}\right)$ measures how the two random velocity vectors at $\mathbf{x}_{0}$ and $\mathbf{x}$ are dependant. We consider an homogeneous, isotropic and stationnary turbulence, in the case of smooth solutions of the NSE. We then perform a expansion of $B\left(\mathbf{x}, \mathbf{x}_{0}\right)$ when $r=\left|\mathbf{x}-\mathbf{x}_{0}\right|$ goes to zero, and we show that the main term in this expansion is entirely characterized by a function $E=E(r)$. We show next that in some inertial range $\left[r_{1}, r_{2}\right]$, $E(r) \sim(\mathscr{E} r)^{\frac{2}{3}}, \mathscr{E}$ being the mean dissipation at $\mathbf{x}_{0}$, which is the law of the $2 / 3$. To do so, we carrefully establish the mathematical concept of similarity principle.

So far we know, this work is the first attempt to give a rigorous proof of the $2 / 3$ law. Nevertheless, the well known law of the $-5 / 3$ about the velocity spectrum, and everything which relates, has attracted a lot of attention in the mathematical literature, see for instance in $[5,8,10,11,12,15,18]$

Ackowledgements. The author thanks Benoit Pinier for a carrefull proofreading of this text. He also thanks Antoine Chambert-Loir for intesting discussions about the algebraic part of this work.

\section{About the Navier-Stokes Equations}

Let $\Omega \subset \mathbb{R}^{3}$ be a smooth domain, $\Gamma=\partial \Omega$ its boundary, $T>0$ a given time, $Q=[0, T] \times \Omega$. In the following $\mathbf{v}=\mathbf{v}(t, \mathbf{x}), p=p(t, \mathbf{x})$ denote the Euler velocity and the pressure of the fluid at any $(t, \mathbf{x}) \in Q$.

A divergence free field $\mathbf{v}_{0}$ being given, the incompressible Navier-Stokes Equations (NSE) 
set in $Q$ with the no slip bounary condition and $\mathbf{v}_{0}$ as initial data are the following:

$$
\left\{\begin{aligned}
\partial_{t} \mathbf{v}+(\mathbf{v} \cdot \nabla) \mathbf{v}-\nabla \cdot(2 \nu D \mathbf{v})+\nabla p & =\mathbf{f} & & \text { in } Q, \\
\nabla \cdot \mathbf{v} & =0 & & \text { in } Q, \\
\mathbf{v} & =0 & & \text { on } \Gamma, \\
\mathbf{v} & =\mathbf{v}_{0} & & \text { at } t=0,
\end{aligned}\right.
$$

where $\nu>0$ is the kinematic viscosity, usually a function of the temperature,

$$
D \mathbf{v}=\frac{1}{2}\left(\nabla \mathbf{v}+\nabla \mathbf{v}^{t}\right),
$$

is the deformation tensor, $\mathbf{f}$ is any external force (gravity, electromagnetic force....). Equation (i) of system (2.1), labeled with (2.1.i), is the momentum equation. Equation (ii) of (2.1), labeled with (2.1.ii), is the mass conservation equation, that expresses in this case the incompressibility of the fluid. Equation (iii) is the boundary condition, namely the no slip condition, whereas (iv) is the initial data. Notice that when $\nu$ is constant, we deduce from the incompressibility condition the identity

$$
\nabla \cdot(2 \nu D \mathbf{v})=\nu \Delta \mathbf{v}
$$

By writing $\mathbf{v}=\left(v_{1}, v_{2}, v_{3}\right)$, the momentum equation (2.1.i) becomes component-by-component:

$$
\partial_{t} v_{i}+v_{j} \frac{\partial v_{i}}{\partial x_{j}}-\frac{\partial}{\partial x_{j}}\left(\nu\left(\frac{\partial v_{i}}{\partial x_{j}}+\frac{\partial v_{j}}{\partial x_{i}}\right)\right)+\frac{\partial p}{\partial x_{i}}=f_{i}
$$

by using the Einstein summation convention. Moreover, the incompressiblity condition (2.1.ii) reads:

$$
\frac{\partial v_{j}}{\partial x_{j}}=0
$$

It is convenient to notice that the incompressibility condition yields

$$
v_{j} \frac{\partial v_{i}}{\partial x_{j}}=\frac{\partial\left(v_{i} v_{j}\right)}{\partial x_{j}},
$$

hence

$$
(\mathbf{v} \cdot \nabla) \mathbf{v}=\nabla \cdot(\mathbf{v} \otimes \mathbf{v})
$$

where $\mathbf{v} \otimes \mathbf{v}=\left(v_{i} v_{j}\right)_{1 \leq i, j \leq 3}$.

We distinguish two types of solutions to the NSE:

1) The "strong" solutions over a small time interval [0, $T_{\max }$ ["à la" Fujita-Kato [17],

2) the "weak" (also turbulent) solutions, global in time, "à la" Leray-Hopf [19, 22]

1) Strong solutions are essentially $C^{1, \alpha}$ over $\left[0, T_{\max }[\times \Omega\right.$, as long as the data are smooth enough. A given $\mathbf{f}$ being fixed, the time $T_{\max }$ only depends on $\nu$ and $\left\|\mathbf{v}_{0}\right\|$ for a suitable norm, and whatever the choice of $\mathbf{v}_{0}$ smooth enough, the corresponding solution is unique, yielding the writing

$$
\mathbf{v}=\mathbf{v}\left(t, \mathbf{x}, \mathbf{v}_{0}\right), \quad p=p\left(t, \mathbf{x}, \mathbf{v}_{0}\right) .
$$

Strong solutions might be globally defined in time over $\left[0, \infty\right.$ [ when the initial data $\mathbf{v}_{0}$ is "small enough", or the viscosity $\nu$ is "large" enough, which means that the flow is rather laminar (see also in $[3,4,6,20]$ ). 
2) Weak solutions are such that the velocity, denoted here by $\mathbf{v}=\mathbf{v}(t)$, is considered as a trajectory into a suitable Hilbert space $H \subset L^{2}(\Omega)^{3}$, and is weakly continuous from $[0, T]$ into $H$, for any $T>0$. This means that any $\boldsymbol{\eta} \in H$ being given, the function $t \rightarrow\langle\mathbf{v}(t), \boldsymbol{\eta}\rangle$ is a continous function of $t$, where $\langle\cdot, \cdot\rangle$ denotes the scalar product in $H$ (see in $[9,14,24,25,30])$.

Whatever the type of solution one considers, it is not known if it develops a singularity in finite time [13], which means that for some $\left(t_{0}, \mathbf{x}_{0}\right)$,

$$
\lim _{\substack{t \rightarrow t_{0} \\ \mathbf{x} \rightarrow \mathbf{x}_{0}}}|\mathbf{v}(t, \mathbf{x})|=\infty
$$

a question studied in $[2,7,23,28]$. Moreover, it is not known if the Leray-Hopf solution is unique or not.

\section{Similarity and Reynolds number}

\subsection{Dimensions}

Each physical field $\psi=\psi(t, \mathbf{x})$ involved in incompressible flows, can be decomposed as

$$
\psi=\ell^{d_{\ell}(\psi)} \tau^{d_{\tau}(\psi)},
$$

where $\tau=\tau(t, \mathbf{x})$ a time field (expressed in seconds) and $\ell=\ell(t, \mathbf{x})$ a length field (expressed in meters). Because of incompressibility, the mass does not play any role in the dimensional analysis carried out in the following. In the expression above,

$$
\mathbb{D}(\psi)=\left(d_{\ell}(\psi), d_{\tau}(\psi)\right) \in \mathbb{Q}^{2},
$$

is the dimension of $\psi$. Notice that in particular, $\mathbb{D}(\mathbf{x})=(1,0), \mathbb{D}(t)=(0,1)$. Any field $\psi$ such that $\mathbb{D}(\psi)=(0,0)$ is said to be dimensionless. We also use the standard notation

$$
[\psi]=\mathcal{L}^{d_{\ell}(\psi)} \mathcal{T}^{d_{\tau}(\psi)},
$$

which is useful in pratical calculations. The following table gives the dimension of the main scalar fields involved in turbulent flows.

\begin{tabular}{|l|c|}
\hline \multicolumn{1}{|c|}{ scalar field } & dimension $\mathbb{D}$ \\
\hline kinematic viscosity $\nu$ & $(2,-1)$ \\
\hline scalar velocity $u$ & $(1,-1)$ \\
\hline pressure per mass density $p$ & $(2,-2)$ \\
\hline kinetic energy per mass density $E=(1 / 2)|\mathbf{v}|^{2}$ & $(2,-2)$ \\
\hline dissipation per mass density $\varepsilon=2 \nu|D \mathbf{v}|^{2}$ & $(2,-3)$ \\
\hline
\end{tabular}

The following table gives the main dimension of the vector and tensor fields involved in turbulent flows.

\begin{tabular}{|l|c|}
\hline \multicolumn{1}{|c|}{ vector and tensor field } & dimension $\mathbb{D}$ \\
\hline \hline velocity $\mathbf{~ v}$ & $(1,-1)$ \\
\hline deformation tensor $D \mathbf{v}$ & $(0,-1)$ \\
\hline vorticity $\boldsymbol{\omega}$ & $(0,-1)$ \\
\hline Source term $\mathbf{f}$, force per mass unit & $(1,-2)$ \\
\hline stress tensor per mass density $(1 / \rho) \boldsymbol{\sigma}$ & $(2,-2)$ \\
\hline
\end{tabular}




\subsection{Lenght-time bases and generalized Reynolds numbers}

Definition 3.1. A length-time basis is a couple

$$
b=(\lambda, \tau),
$$

where $\lambda$ a given constant length and $\tau$ a constant time.

Definition 3.2. Let $\boldsymbol{\psi}=\boldsymbol{\psi}(t, \mathbf{x})$ (constant, scalar, vector, tensor...) be any given field defined on a cylinder $Q=[0, T] \times \Omega$. Let $\boldsymbol{\psi}_{b}$ be the field defined by

$$
\boldsymbol{\psi}_{b}\left(t^{\prime}, \mathbf{x}^{\prime}\right)=\lambda^{-d_{\ell}(\boldsymbol{\psi})} \tau^{-d_{\tau}(\boldsymbol{\psi})} \boldsymbol{\psi}\left(\tau t^{\prime}, \lambda \mathbf{x}^{\prime}\right), \quad\left(t^{\prime}, \mathbf{x}^{\prime}\right) \in Q_{b}=\left[0, \frac{T}{\tau}\right] \times \frac{1}{\lambda} \Omega,
$$

where $t^{\prime}$ and $\mathbf{x}^{\prime}$ are dimensionless. It is easily checked that $\boldsymbol{\psi}_{b}$ is dimensionless. We say that $\boldsymbol{\psi}_{b}=\boldsymbol{\psi}_{b}\left(t^{\prime}, \mathbf{x}^{\prime}\right)$ is the $b$-dimensionless field deduced from $\boldsymbol{\psi}$.

Let $b=(\lambda, \tau)$ be the length-time basis related to a given scale, and

$$
V=\lambda \tau^{-1},
$$

be the convective associated velocity. We assume in what follows that the kinematic viscosity $\nu$ is constant. Therefore, according to formula (3.5), the b-dimensionless field $\nu_{b}$ deduced from $\nu$ is expressed as

$$
\nu_{b}=\lambda^{-2} \tau \nu=\frac{\nu}{V \lambda}
$$

by involving the associated convective velocity $V$ given by (3.6). Let $\operatorname{Re}(b)$ be the dimensionless number defined by

$$
R(b)=\frac{1}{\nu_{b}}=\frac{V \lambda}{\nu}
$$

We observe that $\operatorname{Re}(b)$ is of the same form as the Reynolds number used in classical fluid dynamics, defined as the quotient of the convective forces intensity by the viscous forces intensity, which is why we call $R e(b)$ a generalized Reynolds number.

\section{3 dimensionless form of the NSE}

Lemma 3.1. Let $b=(\lambda, \tau)$ be any length-time basis, $\left(\mathbf{v}_{b}, p_{b}\right)$ be the b-dimensionless field deduced from $(\mathbf{v}, p)$. Then $\left(\mathbf{v}_{b}, p_{b}\right)$ satisfies the following dimensionless NSE,

$$
\left\{\begin{aligned}
\partial_{t^{\prime}} \mathbf{v}_{b}+\left(\mathbf{v}_{b} \cdot \nabla^{\prime}\right) \mathbf{v}_{b}-\nu_{b} \Delta^{\prime} \mathbf{v}_{b}+\nabla^{\prime} p_{b} & =\mathbf{f}_{b} & & \text { in } Q_{b}, \\
\nabla^{\prime} \cdot \mathbf{v}_{b} & =0 & & \text { in } Q_{b}, \\
\mathbf{v}_{b} & =0 & & \text { on } \Gamma_{b}, \\
\mathbf{v}_{b} & =\left(\mathbf{v}_{0}\right)_{b} & & \text { at } t=0 .
\end{aligned}\right.
$$

Proof. It is easily checked that for any field $\boldsymbol{\psi}=\boldsymbol{\psi}(t, \mathbf{x})$ of class $C^{1}$ in time, of class $C^{2}$ in space,

$$
\begin{aligned}
\partial_{t} \boldsymbol{\psi}(t, \mathbf{x}) & =\lambda^{d_{\ell}(\boldsymbol{\psi})} \tau^{d_{\tau}(\boldsymbol{\psi})-1} \partial_{t^{\prime}} \boldsymbol{\psi}_{b}\left(t^{\prime}, \mathbf{x}^{\prime}\right) \\
\nabla \boldsymbol{\psi}(t, \mathbf{x}) & =\lambda^{d_{\ell}(\boldsymbol{\psi})-1} \tau^{d_{\tau}(\boldsymbol{\psi})} \nabla^{\prime} \boldsymbol{\psi}_{b}\left(t^{\prime}, \mathbf{x}^{\prime}\right) \\
\Delta \boldsymbol{\psi}(t, \mathbf{x}) & =\lambda^{d_{\ell}(\boldsymbol{\psi})-2} \tau^{d_{\tau}(\boldsymbol{\psi})} \nabla^{\prime} \boldsymbol{\psi}_{b}\left(t^{\prime}, \mathbf{x}^{\prime}\right)
\end{aligned}
$$


where $(t, \mathbf{x})=\left(\tau t^{\prime}, \lambda \mathbf{x}^{\prime}\right)$. As

$$
d_{\ell}(\mathbf{v})=1, \quad d_{\tau}(\mathbf{v})=-1, \quad d_{\ell}(p)=2, \quad d_{\tau}(p)=1,
$$

we get from (3.5),

$$
\mathbf{v}(t, \mathbf{x})=\lambda \tau^{-1} \mathbf{v}_{b}\left(t^{\prime}, \mathbf{x}^{\prime}\right), \quad p(t, \mathbf{x})=\lambda^{2} \tau^{-2} p_{b}\left(t^{\prime}, \mathbf{x}^{\prime}\right) .
$$

Then (3.9) results from (3.10), (3.11) and (3.12) applied to $\mathbf{v}$ and $p$, combined with (3.13).

\subsection{Similarity}

The pressure in the NSE is defined up to a constant. Therefore, it naturally belongs to quotient spaces. We denote by $\widetilde{p}$ the class of any $p$ in a suitable quotient space, which does not need to be specified here. For $i=1,2$, let us consider:

i) $Q^{(i)}=\left[0, T^{(i)}\right] \times \Omega^{(i)}$ two cylinders,

ii) $\nu^{(i)}$ two kinematical viscosities,

iii) $\mathbf{f}^{(i)}$ two forces per mass unit,

iv) $\mathbf{v}_{0}^{(i)}=\mathbf{v}_{0}^{(i)}(\mathbf{x})$ two velocity fields defined in $\Omega^{(i)}(i=1,2)$.

Definition 3.3. Let $\left(\mathbf{v}^{(i)}, \widetilde{p}^{(i)}\right)$ be two flows in $Q^{(i)}, i=1,2$. We say that these two flows are similar if there exist two length-time bases $b_{1}$ and $b_{2}$, such that

$$
Q_{b_{1}}^{(1)}=Q_{b_{2}}^{(2)}, \quad\left(\mathbf{v}_{b_{1}}^{(1)}, \widetilde{p}_{b_{1}}^{(1)}\right)=\left(\mathbf{v}_{b_{2}}^{(2)}, \widetilde{p}_{b_{2}}^{(2)}\right) .
$$

Let us consider the NSE equations, for $i=1,2$,

$$
\left\{\begin{aligned}
\partial_{t} \mathbf{v}^{(i)}+\left(\mathbf{v}^{(i)} \cdot \nabla\right) \mathbf{v}^{(i)}-\nu^{(i)} \Delta \mathbf{v}^{(i)}+\nabla p^{(i)} & =\mathbf{f}^{(i)} & & \text { in } Q^{(i)}, \\
\nabla \cdot \mathbf{v}^{(i)} & =0 & & \text { in } Q^{(i)}, \\
\mathbf{v}^{(i)} & =0 & & \text { on } \Gamma^{(i)}, \\
\mathbf{v}^{(i)} & =\mathbf{v}_{0}^{(i)} & & \text { at } t=0 .
\end{aligned}\right.
$$

We assume that the data are such that each of these two NSE have a sufficiently smooth solution $\left(\mathbf{v}^{(i)}, \widetilde{p}^{(i)}\right)$. The similarity hypothesis is stated as follows.

Similarity Hypothesis. If there exist two length-time bases $b_{1}$ and $b_{2}$ such that

$$
Q_{b_{1}}^{(1)}=Q_{b_{2}}^{(2)}, \quad\left(\mathbf{v}_{0}^{(1)}\right)_{b_{1}}=\left(\mathbf{v}_{0}^{(1)}\right)_{b_{2}}, \quad \mathbf{f}_{b_{1}}^{(1)}=\mathbf{f}_{b_{2}}^{(2)}, \quad \nu_{b_{1}}=\nu_{b_{2}},
$$

then the two flows $\left(\mathbf{v}^{(i)}, \widetilde{p}^{(i)}\right)$ are similar.

It results from the foregoing that the similarity hypothesis is satisfied if and only the dimensionless form (3.9) of the NSE has a unique solution, which can be made sure for local time solutions "à la Fujita-Kato". Global time similarity remains a mathematical open problem, although it is intensively used in many engineering and environmental applications.

\section{Homogeneous and Isotropic tensor fields}

We assume that $\Omega$ is a convex set and its boundary (if any) is of class $C^{2}$. Moreover, we also assume that $\Omega \neq \emptyset$. We denote by $\mathcal{B}_{0}=\left(\mathbf{e}_{1}, \mathbf{e}_{2}, \mathbf{e}_{3}\right)$ the canonical basis of $\mathbb{R}^{3}$. 


\subsection{Homogeneity}

Definition 4.1. Let $n \geq 1$ and $p \geq 1$ integers, $\mathcal{T}_{p}$ the set of all tensor of order $p$ in $\mathbb{R}^{3}$. We say that $T$ is a $(n, p)$-order tensor field over $\Omega$ if it defines a map from $\Omega^{n+1}$ into $\mathcal{T}_{p}$,

$$
T:\left\{\begin{aligned}
\Omega^{n+1} & \longrightarrow \mathcal{T}_{p}, \\
\left(\mathbf{x}_{0}, \cdots, \mathbf{x}_{n}\right) & \longrightarrow T\left(\mathbf{x}_{0}, \cdots, \mathbf{x}_{n}\right)=\left(T_{i_{1} \cdots i_{p}}\left(\mathbf{x}_{0}, \cdots, \mathbf{x}_{n}\right)\right)_{1 \leq i_{1}, \cdots, i_{p} \leq 3} .
\end{aligned}\right.
$$

Definition 4.2. The $(n, p)$-order tensor field $T$ is said to be homogenous if and only if it is invariant with respect to the translations, which means that $\forall \mathbf{r} \in \mathbb{R}^{3}$ such that $\forall j=0, \cdots, n, \mathbf{x}_{j}+\mathbf{r} \in \Omega$, then $T\left(\mathbf{x}_{0}+\mathbf{r}, \cdots, \mathbf{x}_{n}+\mathbf{r}\right)=T\left(\mathbf{x}_{0}, \cdots, \mathbf{x}_{n}\right)$.

The following result is straightforward.

Proposition 4.1. Let $T$ be an homogenous $(n, p)$-order tensor. Then it only depends on the $n$ vectors $\mathbf{r}_{j}=\mathbf{x}_{j}-\mathbf{x}_{0}, j=1, \cdots, n$.

The proof of Proposition 4.1 is straithforward. According to it, we shall denote any homogeneous tensor $T$ by $T=T\left(\mathbf{r}_{1}, \cdots, \mathbf{r}_{n}\right)$.

It is worth noting that all this makes sense when the $\mathbf{r}_{j}$ 's belong to the set

$$
\begin{aligned}
\boldsymbol{\eta}_{n}(\Omega)=\left\{P_{n}=\left(\mathbf{r}_{1}, \cdots,\right.\right. & \left.\mathbf{r}_{n}\right) \in \mathbb{R}^{3 n}, \\
& \left.\exists \mathbf{x}_{0} \in \Omega \text { such that } \forall j=1, \cdots, n, \mathbf{x}_{0}+\mathbf{r}_{j} \in \Omega\right\},
\end{aligned}
$$

which is not an empty set. Indeed, as $\Omega \neq \emptyset$, for some $\mathbf{x}_{0} \in \Omega$ and $r_{0}>0, B\left(\mathbf{x}_{0}, r_{0}\right) \subset$ $\Omega \subset \Omega$. Therefore,

$$
\left\{P_{n}=\left(\mathbf{r}_{1}, \cdots, \mathbf{r}_{n}\right) \in \mathbb{R}^{3 n},\left|\mathbf{r}_{j}\right|<r_{0}, j=1, \ldots, n\right\} \subset \boldsymbol{\eta}_{n}(\Omega) .
$$

From now on, we shall deal with $\boldsymbol{\eta}_{n}(\Omega)$. For example, an homogeneous $(1,1)$-order tensor $T=T(\mathbf{r})=\left(T_{1}(\mathbf{r}), T_{2}(\mathbf{r}), T_{3}(\mathbf{r})\right)$ is a vecteur field defined over $\boldsymbol{\eta}_{1}(\Omega)$. Furthermore an homogeneous $(1,2)$-order tensor can be viewed as a matrix

$$
T=T(\mathbf{r})=\left(\begin{array}{ll}
T_{11}(\mathbf{r}) & T_{12}(\mathbf{r}) \\
T_{21}(\mathbf{r}) & T_{22}(\mathbf{r})
\end{array}\right) .
$$

Throughout the following, we shall write $P_{n}=\left(\mathbf{r}_{1}, \cdots, \mathbf{r}_{n}\right) \in \boldsymbol{\eta}_{n}(\Omega)$.

\subsection{Dual action}

Any $(n, p)$-order homogeneous tensor field $T=T\left(P_{n}\right)$ performs a dual action over $\mathbb{R}^{3 p}$ as follows. Let $A_{p}=\left(\mathbf{a}_{1}, \mathbf{a}_{2}, \cdots, \mathbf{a}_{p}\right) \in \mathbb{R}^{3 p}=\mathbb{R}^{3} \times \cdots \times \mathbb{R}^{3}$. We set $\mathbf{a}_{i}=\left(a_{i 1}, a_{i 2}, a_{i 3}\right)$. It is therefore natural to define the dual action of $T\left(P_{n}\right)$ at each $P_{n} \in \boldsymbol{\eta}_{n}(\Omega)$ over $\mathbb{R}^{3 p}$ by the expression

$$
\left[T\left(P_{n}\right), A_{p}\right]=T_{i_{1} \cdots i_{p}}\left(P_{n}\right) a_{i_{1} 1} \cdots a_{i_{p} p},
$$

that we also could write

$$
\left[T\left(P_{n}\right), A_{p}\right]=T\left(P_{n}\right): \mathbf{a}_{1} \otimes \mathbf{a}_{2} \otimes \ldots . \otimes \mathbf{a}_{n},
$$

where ": " stands for the contracted tensor product, " $\otimes$ " the tensor product.

For example, when $T=T(\mathbf{r})=\left(T_{1}(\mathbf{r}), T_{2}(\mathbf{r}), T_{3}(\mathbf{r})\right)$ is a $(1,1)$-order tensor, this dual action is the standard scalar product on $\mathbb{R}^{3}$,

$$
[T(\mathbf{r}), \mathbf{a}]=\left(T\left(P_{n}\right), \mathbf{a}\right)=T_{i}\left(P_{n}\right) a_{i} .
$$

When $T(\mathbf{r})=\left(T_{i j}(\mathbf{r})\right)_{1 \leq i j \leq 3}$ is a $(1,2)$-order tensor, then

$$
[T(\mathbf{r}),(\mathbf{a}, \mathbf{b})]=T_{i j}(\mathbf{r}) a_{i} b_{j}=(T(\mathbf{r}) \cdot \mathbf{b}, \mathbf{a}),
$$

where $T(\mathbf{r}) \cdot \mathbf{b}$ denotes the product of the matrix $T(\mathbf{r})$ with the vector $\mathbf{b}$. 


\subsection{Isotropy}

Usually, a tensor field $T$ is said to be isotropic if it exerts the same action regardless of the direction, a notion that remains to be rigorously defined. To do so, let us introduce $\mathrm{O}_{3}(\mathbb{R})$ the orthogonal group, characterised by:

$$
Q \in O_{3}(\mathbb{R}) \text { if and only if } Q Q^{t}=\mathrm{I}_{3},
$$

where $\mathrm{I}_{3}$ denotes the identity of $\mathbb{R}^{3}, Q^{t}$ the transpose of $Q$. To well define the notion of isotropy, we also will need the set

$$
\begin{aligned}
\iota_{n}(\Omega)=\left\{P_{n}=\left(\mathbf{r}_{1}, \ldots,\right.\right. & \left.\mathbf{r}_{n}\right) \in \boldsymbol{\eta}_{n}(\Omega), \\
& \left.\forall Q \in O_{3}(\mathbb{R}), Q P_{n}=\left(Q \mathbf{r}_{1}, \ldots, Q \mathbf{r}_{n}\right) \in \boldsymbol{\eta}_{n}(\Omega)\right\} .
\end{aligned}
$$

It is easily checked that $\boldsymbol{\iota}_{n}(\Omega) \neq \emptyset$. Finally, let $A_{p}=\left(\mathbf{a}_{1}, \mathbf{a}_{2}, \cdots, \mathbf{a}_{p}\right) \in \mathbb{R}^{3 p}, Q \in O_{3}(\mathbb{R})$, and let $Q A_{p}$ be specified by

$$
Q A_{p}=\left(Q \mathbf{a}_{1}, Q \mathbf{a}_{2}, \cdots, Q \mathbf{a}_{p}\right) \in \mathbb{R}^{3 p} .
$$

Definition 4.3. Let $T$ be an homogeneous $(n, p)$-order tensor field over $\Omega$. The tensor $T$ is said to be isotropic if and only if,

$$
\forall P_{n} \in \iota_{n}(\Omega), \quad \forall A_{p} \in \mathbb{R}^{3 p}, \quad \forall Q \in O_{3}(\mathbb{R}), \quad\left[T\left(Q P_{n}\right), Q A_{p}\right]=\left[T\left(P_{n}\right), A_{p}\right] .
$$

\subsection{1-1 isotropic tensor fields}

We aim in this section to caracterize 1-1 isotropic tensors. The main result is that the unique 1-1 isotropic tensor, which is smoth and has a free divergence, is the null tensor. In the following, any $\mathbf{r}=\left(r_{1}, r_{2}, r_{3}\right) \in \mathbb{R}^{3}$ being given, we set $r=|\mathbf{r}|=\left(r_{1}^{2}+r_{2}^{2}+r_{3}^{2}\right)^{\frac{1}{2}}$.

Theorem 4.1. Let $\mathbf{w}=\mathbf{w}(\mathbf{r})=\left(w_{1}(\mathbf{r}), w_{2}(\mathbf{r}), w_{3}(\mathbf{r})\right)$ be a 1-1 order isotropic tensor field. Then there exists a function $a=a(r)$ such that

$$
\forall \mathbf{r} \in \iota_{1}(\Omega), \quad \mathbf{w}(\mathbf{r})=a(r) \frac{\mathbf{r}}{r} .
$$

Assume in addition that $\mathbf{w}$ is differentiable over $\iota_{1}(\Omega) \backslash B\left(0, r_{0}\right)$ for some $r_{0}>0$, and is incompressible with respect to $\mathbf{r}$. Then if $\mathbf{w}$ is not identically equal to zero, there exists a constant $K$ such that

$$
\forall \mathbf{r} \in \iota_{1}(\Omega), \quad \mathbf{w}(\mathbf{r})=K \frac{\mathbf{r}}{r}
$$

for all $\mathbf{r} \in \iota_{1}(\Omega) \backslash B\left(0, r_{0}\right)$.

Proof. Let $\mathbf{a} \in \mathbb{R}^{3}, Q \in O_{3}(\mathbb{R})$. Remember that $[\mathbf{w}(\mathbf{r}), \mathbf{a}]=(\mathbf{w}(\mathbf{r}), \mathbf{a})$ is the standard scalar product in $\mathbb{R}^{3}$. From the isotropy assumption and the relation $Q^{t}=Q^{-1}$ because $Q \in O_{3}(\mathbb{R})$, we get the equalities

$$
[\mathbf{w}(Q \mathbf{r}), Q \mathbf{a}]=(\mathbf{w}(Q \mathbf{r}), Q \mathbf{a})=\left(Q^{-1} \mathbf{w}(Q \mathbf{r}), \mathbf{a}\right)=(\mathbf{w}(\mathbf{r}), \mathbf{a})=[\mathbf{w}(\mathbf{r}), \mathbf{a}] .
$$

As this equality holds $\forall \mathbf{a} \in \mathbb{R}^{3}$, the isotropy of $\mathbf{w}$ yields

$$
\forall Q \in O_{3}(\mathbb{R}), \quad \forall \mathbf{r} \in \iota_{1}(\Omega), \quad Q^{-1} \mathbf{w}(Q \mathbf{r})=\mathbf{w}(\mathbf{r}) .
$$


Let $\mathbf{r}=r \mathbf{e}_{1}$, with $r \in\left[0, r_{0}\left[\right.\right.$ such that $r \mathbf{e}_{1} \in \boldsymbol{\iota}_{1}(\Omega)$. Let $Q \in O_{3}(\mathbb{R})$ partitioned into 4 blocks of the form

$$
Q=\left(\begin{array}{cl}
1 & 0 \\
0 & P
\end{array}\right), \quad P \in O_{2}(\mathbb{R})
$$

It is easily checked that $Q\left(\mathbf{e}_{1}\right)=\mathbf{e}_{1}$. Therefore, we deduce from (4.8)

$$
Q^{-1} \mathbf{w}\left(r \mathbf{e}_{1}\right)=\mathbf{w}\left(r \mathbf{e}_{1}\right) .
$$

Let us write $\mathbf{w}\left(r \mathbf{e}_{1}\right)=(a(r), b(r), c(r))^{t}$, that we insert in (4.10), which yields

$$
Q \mathbf{w}\left(r \mathbf{e}_{1}\right)=\left(\begin{array}{ll}
1 & 0 \\
0 & P
\end{array}\right)\left(\begin{array}{c}
a(r) \\
b(r) \\
c(r)
\end{array}\right)=\left(\begin{array}{c}
a(r) \\
P\left(\begin{array}{c}
b(r) \\
c(r)
\end{array}\right)
\end{array}\right)=\left(\begin{array}{c}
a(r) \\
b(r) \\
c(r)
\end{array}\right),
$$

leading to

$$
P\left(\begin{array}{l}
b(r) \\
c(r)
\end{array}\right)=\left(\begin{array}{c}
b(r) \\
c(r)
\end{array}\right)
$$

which holds $\forall P \in O_{2}(\mathbb{R})$. Therefore, $b(r)=c(r)=0$. Consequently,

$$
\mathbf{w}\left(r \mathbf{e}_{1}\right)=a(r) \mathbf{e}_{1} .
$$

Let $\mathbf{r} \in \boldsymbol{\iota}_{1}(\Omega)$. There exists $Q \in O_{3}(\mathbb{R})$ such that

$$
\frac{\mathbf{r}}{r}=Q\left(\mathbf{e}_{1}\right) .
$$

We deduce from (4.8) that

$$
\mathbf{w}(\mathbf{r})=Q \mathbf{w}\left(r \mathbf{e}_{1}\right)=a(r) \frac{\mathbf{r}}{r},
$$

which concludes the proof of (4.6).

We are left with proving (4.7). Assume that $a$ is of class $C^{1}$ over $\left[r_{0}, r_{1}\left[\right.\right.$ for some $0<r_{0}<$ $r_{1}$, and that $\mathbf{w}$ is incompressible over $\iota_{1}(\Omega) \backslash B\left(0, r_{0}\right)$. For simplicity, we put $\alpha(r)=a(r) / r$. In this formalism, $w_{i}=\alpha(r) r_{i}$. Since $\partial_{i} r=r_{i} / r$, we deduce from the incompressibility assumption the differential equation

$$
\nabla \cdot \mathbf{w}=r \alpha^{\prime}(r)+\alpha(r)=0,
$$

Integrating (4.12) over $\left[r_{0}, r_{1}[\right.$ yields

$$
\alpha(r)=\frac{K}{r}
$$

hence the result.

The following corollary is straightforward.

Corollary 1. Let $\mathbf{w}=\mathbf{w}(\mathbf{r})=\left(w_{1}(\mathbf{r}), w_{2}(\mathbf{r}), w_{3}(\mathbf{r})\right)$ be a 1-1 order isotropic tensor field of class $C^{1}$ over $\iota_{1}$, and incompressible over $\boldsymbol{\iota}_{1}$. Then $\mathbf{w}$ is identically equal to 0 . 


\section{$4.5 \quad 1-2$ isotropic tensor fields}

This section is devoted to the characterization of 1-2 isotropic tensor fields.

Theorem 4.2. Let $B(\mathbf{r})=\left(B_{i j}(\mathbf{r})\right)_{1 \leq i, j \leq 3}$ be a 1-2 order isotropic tensor field. Then there exists a function $B_{d}=B_{d}(r)$ and a function $B_{n}=B_{n}(r)$ such that $\forall \mathbf{r} \in \iota_{1}(\Omega)$,

$$
B(\mathbf{r})=\left(B_{d}(r)-B_{n}(r)\right) \frac{\mathbf{r} \otimes \mathbf{r}}{r^{2}}+B_{n}(r) \mathrm{I}_{3} .
$$

Proof. Let $(\mathbf{a}, \mathbf{b}) \in\left(\mathbb{R}^{3}\right)^{2}$ be any vector. We already know that

$$
[B(\mathbf{r}),(\mathbf{a}, \mathbf{b})]=B_{i j}(\mathbf{r}) a_{i} b_{j}=(B(\mathbf{r}) \cdot \mathbf{b}, \mathbf{a}),
$$

The isotropy assumption yields

$$
\forall Q \in O_{3}(\mathbb{R}), \quad(B(Q \mathbf{r}) \cdot Q \mathbf{b}, Q \mathbf{a})=(B(\mathbf{r}) \cdot \mathbf{b}, \mathbf{a})=\left(Q^{-1} B(Q \mathbf{r}) Q \mathbf{b}, \mathbf{a}\right),
$$

where we have used $Q^{t}=Q^{-1}$. Since this relation holds for all $(\mathbf{a}, \mathbf{b}) \in \mathbb{R}^{3} \times \mathbb{R}^{3}$, the isotropy assumption leads to

$$
\forall \mathbf{r} \in \iota_{1}(\Omega), \quad Q^{-1} B(Q \mathbf{r}) Q=B(\mathbf{r}),
$$

or alternatively

$$
\forall \mathbf{r} \in \iota_{1}(\Omega), \quad B(Q \mathbf{r}) Q=B R(\mathbf{r}),
$$

We consider again $\mathbf{r}=r \mathbf{e}_{1}$ and $Q$ as (4.9), so that $Q\left(\mathbf{e}_{1}\right)=\mathbf{e}_{1}$. We write

$$
B\left(r \mathbf{e}_{1}\right)=\left(\begin{array}{ll}
B_{d}(r) & \mathbf{w}^{t} \\
\mathbf{v} & H
\end{array}\right),
$$

where $B_{d}(r)$ is a scalar function, $\mathbf{v}$ and $\mathbf{w}$ vectors in $\mathbb{R}^{2}$ and $H$ a $2 \times 2$ matrix, all depending on $r$. We deduce from (4.17),

$$
Q B\left(r \mathbf{e}_{1}\right)=\left(\begin{array}{ll}
B_{d}(r) & \mathbf{w}^{t} \\
P \mathbf{v} & P H
\end{array}\right)=B\left(r \mathbf{e}_{1}\right) Q=\left(\begin{array}{ll}
B_{d}(r) & \mathbf{w}^{t} P \\
\mathbf{v} & H P
\end{array}\right),
$$

Therefore,

$$
\forall P \in O_{2}(\mathbb{R}), \quad \forall(\mathbf{v}, \mathbf{w}) \in \mathbb{R}^{2} \times \mathbb{R}^{2}, \quad P \mathbf{v}=\mathbf{v}, \quad \mathbf{w}^{t} P=\mathbf{w}^{t}, \quad P H=H P,
$$

which yields $\mathbf{v}=\mathbf{w}=0$. Moreover, we know from standard alegbra that only scalar matrices commute with all matrices in $O_{2}(\mathbb{R})$, which leads to $H=B_{n}(r) \mathrm{I}_{2}$, for some scalar function $B_{n}(r)$. Summerizing, we have

$$
B\left(r \mathbf{e}_{1}\right)=\left(\begin{array}{lll}
B_{d}(r) & 0 & 0 \\
0 & B_{n}(r) & 0 \\
0 & 0 & B_{n}(r)
\end{array}\right)=\left(B_{d}(r)-B_{n}(r)\right) \mathbf{e}_{1} \otimes \mathbf{e}_{1}+B_{n}(r) \mathrm{I}_{3} .
$$

Formula (4.13) results from (4.16) combined with (4.21). Indeed, let $\mathbf{r} \in \iota_{1}(\Omega)$. There exists $Q \in O_{3}(\mathbb{R})$ such that $\mathbf{r}=r Q\left(\mathbf{e}_{1}\right)$, and we notice that

$$
Q\left(\mathbf{e}_{1} \otimes \mathbf{e}_{1}\right) Q^{-1}=\left(Q \mathbf{e}_{1}\right) \otimes\left(Q \mathbf{e}_{1}\right)=\frac{\mathbf{r} \otimes \mathbf{r}}{r^{2}},
$$

because $Q^{t}=Q^{-1}$.

Remark 4.1. By writting $\mathbf{r}=\left(r_{1}, r_{2}, r_{3}\right), B=\left(B_{i j}\right)_{1 \leq i j \leq 3}$, we deduce from (4.21) the relations

$$
\begin{aligned}
& B_{11}(r, 0,0)=B_{d}(r), \\
& B_{22}(r, 0,0)=B_{33}(r, 0,0)=B_{n}(r), \\
& B_{i j}(r, 0,0)=0, \quad \forall i \neq j .
\end{aligned}
$$




\section{Homeneous and isotropic turbulence}

We will have define what is an homogeneous and isotropic turbulence by the end of this section.

\subsection{Statistics and Reynolds Stress}

Following [5], chapter 4 , we consider a compact set $\mathbb{K}$ of $C^{2, \alpha}$ divergence free vector field on $\Omega$. We also know from [5] that there exists a probablity measure $\mu$ defined on $\mathbb{K}$. Moreover, there exists $\delta T>0$ such that for all $\mathbf{v}_{0} \in \mathbb{K}$, the NSE have a unique strong solution $\mathbf{v}=\mathbf{v}(t, \mathbf{x})$ for $t \in[0, \delta T]$, such that $\mathbf{v}(0, \mathbf{x})=\mathbf{v}_{0}(\mathbf{x})$. By setting $\mathbf{v}=\mathbf{v}\left(t, \mathbf{x}, \mathbf{v}_{0}\right)$, the velocity becomes a random variable, which allows to consider the mean field

$$
\overline{\mathbf{v}}(t, \mathbf{x})=E_{\mu}(\mathbf{v})=\int_{\mathbb{K}} \mathbf{v}\left(t, \mathbf{x}, \mathbf{v}_{0}\right) d \mu\left(\mathbf{v}_{0}\right),
$$

where in addition

$$
\overline{\mathbf{v}}(0, \mathbf{x})=\overline{\mathbf{v}_{0}}(\mathbf{x})=\int_{\mathbb{K}} \mathbf{v}_{0}(\mathbf{x}) d \mu\left(\mathbf{v}_{0}\right) .
$$

We will also consider the mean pressure:

$$
\bar{p}(t, \mathbf{x})=\int_{\mathbb{K}} p\left(t, \mathbf{x}, \mathbf{v}_{0}\right) d \mu\left(\mathbf{v}_{0}\right) .
$$

We can decompose $(\mathbf{v}, p)$ as follows:

$$
\mathbf{v}=\overline{\mathbf{v}}+\mathbf{v}^{\prime}, \quad p=\bar{p}+p^{\prime},
$$

which is known as the Reynolds decomposition. The fields $\mathbf{v}^{\prime}$ and $p^{\prime}$ are the fluctuations. We deduce from standard results in analysis the following Reynolds rules:

$$
\begin{aligned}
\overline{\partial_{t} \mathbf{v}\left(t, \mathbf{x}, \mathbf{v}_{0}\right)} & =\partial_{t} \overline{\mathbf{v}}(t, \mathbf{x}), \\
\overline{\nabla \mathbf{v}\left(t, \mathbf{x}, \mathbf{v}_{0}\right)} & =\nabla \overline{\mathbf{v}}(t, \mathbf{x}), \\
\overline{\nabla p\left(t, \mathbf{x}, \mathbf{v}_{0}\right)} & =\nabla \bar{p}(t, \mathbf{x}) .
\end{aligned}
$$

Moreover, by noting that $\overline{\mathbf{v}}=\overline{\mathbf{v}}$ and $\overline{\bar{p}}=\bar{p}$, it easily checked that:

Lemma 5.1. The fluctuation's mean vanishes, i.e.

$$
\forall(t, \mathbf{x}) \in Q_{m}, \quad \overline{\mathbf{v}^{\prime}\left(t, \mathbf{x}, \mathbf{v}_{0}\right)}=0, \quad \overline{p^{\prime}\left(t, \mathbf{x}, \mathbf{v}_{0}\right)}=0 .
$$

Therefore, applying Lemma 5.1 and taking the mean of the NSE yields the PDE system

$$
\left\{\begin{aligned}
\partial_{t} \overline{\mathbf{v}}+(\overline{\mathbf{v}} \cdot \nabla) \overline{\mathbf{v}}-\nu \Delta \overline{\mathbf{v}}+\nabla \bar{p} & =-\nabla \cdot \boldsymbol{\sigma}^{(\mathrm{R})}+\mathbf{f} & & \text { in } Q_{m}, \\
\nabla \cdot \overline{\mathbf{v}} & =0 & & \text { in } Q_{m}, \\
\overline{\mathbf{v}} & =0 & & \text { on } \Gamma, \\
\overline{\mathbf{v}} & =\overline{\mathbf{v}_{0}} & & \text { at } t=0,
\end{aligned}\right.
$$

where $Q_{m}=[0, \delta T] \times \Omega$, and by assuming that $\mathbf{f}$ is a given constant field. In the equation above,

$$
\boldsymbol{\sigma}^{(\mathrm{R})}=\overline{\mathbf{v}^{\prime} \otimes \mathbf{v}^{\prime}}
$$

is the Reynolds stress. A thorough presentation of this process can be found in [5]). 
Remark 5.1. Any field $\boldsymbol{\psi}$ related to the flow still satisfies the reynolds rules (5.5) and (5.6)

Remark 5.2. The average process introduced above does not affect the dimension, in the sense that

$$
\mathbb{D}(\psi)=\mathbb{D}(\bar{\psi}),
$$

for each field $\psi$ related to the flow.

\subsection{Correlation tensors, main assumptions}

Following Prandtl [26], we consider a ball of fluid $\delta V \subset \Omega$, whose diameter is equal to $\ell$ and which is centered at $\mathbf{x}_{0}, \ell$ being the Prandtl mixing length at $\mathbf{x}_{0}$. Following Kolmogorov [21] we consider for $(t, \mathbf{x}) \in[0, \delta T] \times \delta V$,

$$
\mathbf{w}(t, \mathbf{x})=\mathbf{v}(t, \mathbf{x})-\mathbf{v}\left(t, \mathbf{x}_{0}\right), \quad \mathbf{w}=\left(w_{1}, w_{2}, w_{3}\right) .
$$

The general n-order correlation tensor $T^{(n)}=T^{(n)}\left(t, \mathbf{x}_{0}, \mathbf{r}_{1}, \cdots, \mathbf{r}_{n}\right)$ is specified component by component by the expression

$$
T_{i_{1} \cdots i_{n}}^{(n)}\left(t, \mathbf{x}_{0}, \mathbf{r}_{1}, \cdots, \mathbf{r}_{n}\right)=\overline{w_{i_{1}}\left(t, \mathbf{x}_{0}+\mathbf{r}_{1}\right) \cdots w_{i_{n}}\left(t, \mathbf{x}_{0}+\mathbf{r}_{n}\right)},
$$

We assume that in $\delta V$ the turbulence is i) stationnary, ii) homogeneous, which is reflected by:

i) The correlation tensors are invariant under time translation, which yields in this case that they do not depend on $t$,

ii) The correlation tensors are invariant under spatial translations, in the sense that

$$
\forall \mathbf{r}, \quad T^{(n)}\left(t, \mathbf{x}_{0}, \mathbf{r}_{1}+\mathbf{r}, \cdots, \mathbf{r}_{n}+\mathbf{r}\right)=T^{(n)}\left(t, \mathbf{x}_{0}, \mathbf{r}_{1}, \cdots, \mathbf{r}_{n}\right) .
$$

so far the quantities above are well defined.

Therefore, the correlation tensor does not depend on $t$ nor on $\mathbf{x}_{0}$, that might have been chosen anywhere in $\delta V$. Consequently, we can write $T^{(n)}=T^{(n)}\left(\mathbf{r}_{1}, \cdots, \mathbf{r}_{n}\right)$, for $\left(\mathbf{r}_{1}, \cdots, \mathbf{r}_{n}\right) \in$ $\boldsymbol{\eta}_{n}(\delta V)$ (see Definition 4.1).

Remark 5.3. We could also have set

$$
T_{i_{1} \cdots i_{n}}^{(n)}\left(t_{1}, \cdots, t_{n}, \mathbf{x}_{0}, \mathbf{r}_{1}, \cdots, \mathbf{r}_{n}\right)=\overline{w_{i_{1}}\left(t_{1}, \mathbf{x}_{0}+\mathbf{r}_{1}\right) \cdots w_{i_{n}}\left(t_{n}, \mathbf{x}_{0}+\mathbf{r}_{n}\right)},
$$

which is the most general correlation tensor that can be considered, the study of which is out the scope of this text.

Finally, we assume that the turbulence is isotropic in $\delta V$, meaning that all correlation tensors are isotropic (see Definition 4.3).

Remark 5.4. Notice that $\overline{\mathbf{w}}=\overline{\mathbf{w}}(\mathbf{r})$ is a 1-1 divergence free tensor, which is of class $C^{1, \alpha}$ over $[0, \delta T] \times \delta V$ and isotropic. Therefore, according to Corollary $1, \overline{\mathbf{w}}=0$. The main consequence is that the mean velocity field $\overline{\mathbf{v}}$ is constant in $[0, \delta T] \times \delta V$. Consequently, the NSE (5.8) reduces to:

$$
\nabla \cdot \boldsymbol{\sigma}^{(\mathrm{R})}+\nabla \bar{p}=\mathbf{f}
$$


Definition 5.1. We say that the turbulence is homogeneous and isotropic in $\delta V$ if all the correlation tensors are homogeneous and isotropic (see Definition 4.3).

Remark 5.5. It is implicitely assumed in [21] that the velocity is a random vector field that has a distribution function, although the probabilistic space is not specified. This crucial point is far to be obvious, which remains an open issue in our framework. However, if this claim is true, then the correlation tensor can also be considered as the momentum of this distribution function.

Throughout the rest of this text, we shall assume that the turbulence is homogeneous and isotropic. It must be stressed that this assumption may depend on the choice of the initial data set $\mathbb{K}$ and the probability measure $\mu$, and we do not know wether there exists $\mathbb{K}$ and $\mu$ such that homogeneity and isotropy is hold.

\section{Covariance matrix and the law of the $2 / 3$}

\subsection{Asymptotic expansion of the covariance matrix}

We focus in this subsection on the 1-2 energy tensor, as first introduced in [21, 29]:

$$
B(\mathbf{r})=T^{(2)}(\mathbf{r}, \mathbf{r}),
$$

that we write for simplicity $B_{i j}(\mathbf{r})=\overline{w_{i}(\mathbf{r}) w_{j}(\mathbf{r})}$. This matrix, or tensor, is the covariance matrix of the vector fields $\mathbf{v}$ at $\mathbf{x}_{0}$ and at any next point $\mathbf{x}$. Roughly speaking, it measures how the velocity at a given point is correlated to the velocity at a next point. In the case the velocities follow gaussian laws, the velocities are independant random fields if and only if $B$ is the null matrix, which may be not happend in the non gaussian case.

The main result of this section is Theorem 6.1, which somehow proves relation (25) stated at the end of [21].

As we have assumed that the turbulence is homogeneous and isotropic in $\delta V$, we deduce from Theorem 4.2 that there exists two scalar functions $B_{d}(r)$ and $B_{n}(r)$ such that:

$$
B(\mathbf{r})=\left(B_{d}(r)-B_{n}(r)\right) \frac{\mathbf{r} \otimes \mathbf{r}}{r^{2}}+B_{n}(r) \mathrm{I}_{3} .
$$

Moreover, we also know from Remark 4.1 that

$$
\begin{aligned}
& B_{d}(r)=\overline{\left|w_{1}(r, 0,0)\right|^{2}}, \\
& B_{n}(r)=\overline{\left|w_{2}(r, 0,0)\right|^{2}}=\overline{\left|w_{3}(r, 0,0)\right|^{2}}, \\
& \forall i \neq j, \quad \overline{w_{i}(r, 0,0) w_{j}(r, 0,0)}=0 .
\end{aligned}
$$

Lemma 6.1. The following holds:

$$
\begin{aligned}
& B_{d}(0)=B_{n}(0)=0, \\
& B_{d}^{\prime}(0)=B_{n}^{\prime}(0)=0,
\end{aligned}
$$

Proof. Equality (6.6) results from (6.3) and (6.4) combined with $\mathbf{w}(0,0,0)=0$. To check (6.7), we use the Reynolds rule (5.6), and we get

$$
B_{d}^{\prime}(r)=2 \overline{\frac{\partial w_{1}(r, 0,0)}{\partial r_{1}} w_{1}(r, 0,0)},
$$

hence the equality $B_{d}^{\prime}(0)$ because $\mathbf{w}(0,0,0)=0$. The proof of $B_{n}^{\prime}(0)=0$ is similar. 
Theorem 6.1. Assume that the mean pressure gradient is constant inside $\delta V$. Then there exists a $C^{1}$ scalar function $E=E(r)$ such that $E(0)=E^{\prime}(0)=0$ and such that

$$
B(\mathbf{r})=E(r) \frac{\mathbf{r} \otimes \mathbf{r}}{r^{2}}-\frac{3}{2} E(r) \mathrm{I}_{3}+o\left(r^{3}\right) .
$$

Proof. We denote by $\nabla_{\mathbf{x}}$ and $\nabla_{\mathbf{r}}$ the gradient with respect to $\mathbf{x}$ and $\mathbf{r}$ respectively. The proof is divided into 3 steps. In the first step, we expand $\nabla_{\mathbf{r}} \cdot B(\mathbf{r})$ from (6.2) by using Lemma 6.1. In the second step we expand $\nabla_{\mathbf{r}} \cdot B(\mathbf{r})$ from the NSE. In the last step, we combine the two expensions and get the conclusion.

Step 1. We deduce from (6.2) and a standard calculation the relation:

$$
\nabla_{\mathbf{r}} \cdot B(\mathbf{r})=\frac{\mathbf{r}}{r^{2}}\left(r B_{d}^{\prime}(r)+\left(B_{d}(r)-B_{n}(r)\right) .\right.
$$

Identities (6.6) and (6.7) yield the Taylor expansion

$$
\begin{aligned}
& B_{d}(r)=\alpha_{d} r^{2}+r^{3} \varepsilon_{d}(r), \\
& B_{n}(r)=\alpha_{n} r^{2}+r^{3} \varepsilon_{n}(r),
\end{aligned}
$$

where $\alpha_{n}$ and $\alpha_{d}$ are constant coefficients, $\varepsilon_{d}$ and $\varepsilon_{n}$ are smooth bounded functions, the derivative of which are bounded. Moreover, by (6.3) and (6.4) we get $\alpha_{n} \geq 0$ and $\alpha_{d} \geq 0$. Therefore, equality (6.9) becomes

$$
\nabla_{\mathbf{r}} \cdot B(\mathbf{r})=\left(3 \alpha_{d}-\alpha_{n}\right) \mathbf{r}+r^{2} \varepsilon(\mathbf{r})
$$

for some smooth bounded function $\varepsilon$ that does not need to be specified.

Step 2. We start from $B_{i j}(\mathbf{r})=\overline{w_{i}(\mathbf{r}) w_{j}(\mathbf{r})}$ combined with $w_{i}(\mathbf{r})=v_{i}\left(\mathbf{x}_{0}+\mathbf{r}\right)-v_{i}\left(\mathbf{x}_{0}\right)$, which yields the decomposition

$$
B_{i j}(\mathbf{r})=M_{i j}(\mathbf{r})-N_{i j}(\mathbf{r})-P_{i j}(\mathbf{r})+Q_{i j}(\mathbf{r}),
$$

where

$$
\left\{\begin{aligned}
M_{i j}(\mathbf{r}) & =\overline{v_{i}\left(\mathbf{x}_{0}+\mathbf{r}\right) v_{j}\left(\mathbf{x}_{0}+\mathbf{r}\right)}, \\
N_{i j}(\mathbf{r}) & =\overline{v_{i}\left(\mathbf{x}_{0}\right) v_{j}\left(\mathbf{x}_{0}+\mathbf{r}\right)}, \\
P_{i j}(\mathbf{r}) & =\frac{v_{i}\left(\mathbf{x}_{0}+\mathbf{r}\right) v_{j}\left(\mathbf{x}_{0}\right)}{Q_{i j}(\mathbf{r})}=
\end{aligned}\right.
$$

In the following, we set

$$
\mathbf{M}=\left(M_{i j}\right)_{1 \leq i j \leq 3}, \quad \mathbf{N}=\left(N_{i j}\right)_{1 \leq i j \leq 3}, \quad \mathbf{P}=\left(P_{i j}\right)_{1 \leq i j \leq 3}, \quad \mathbf{Q}=\left(Q_{i j}\right)_{1 \leq i j \leq 3},
$$

so that decomposition (6.12) becomes

$$
B=\mathbf{M}-\mathbf{N}-\mathbf{P}+\mathbf{Q} .
$$

We will now use the Reynolds decomposition $\mathbf{v}=\overline{\mathbf{v}}+\overline{\mathbf{v}}^{\prime}$, and study each term in the r.h.s of (6.12) one after each other. We recall that $\boldsymbol{\sigma}^{(\mathrm{R})}=\left(\sigma_{i j}\right)_{1 \leq i j \leq 3}$ is the Reynolds stress. (i) - We easily obtain

$$
M_{i j}(\mathbf{r})=\overline{v_{i}\left(\mathbf{x}_{0}+\mathbf{r}\right)} \cdot \overline{v_{j}\left(\mathbf{x}_{0}+\mathbf{r}\right)}+\overline{v_{i}^{\prime}\left(\mathbf{x}_{0}+\frac{\mathbf{r}) \overline{v_{j}\left(\mathbf{x}_{0}+\mathbf{r}\right)}}{\overline{v_{i}\left(\mathbf{x}_{0}+\mathbf{r}\right)} v_{j}^{\prime}\left(\mathbf{x}_{0}+\mathbf{r}\right)}+\sigma_{i j}\left(\mathbf{x}_{0}+\mathbf{r}\right) .\right.}
$$


As $\overline{\mathbf{v}^{\prime}}=0$, the second and third term in the r.h.s of this equality vanish, hence

$$
M_{i j}(\mathbf{r})=\overline{v_{i}\left(\mathbf{x}_{0}+\mathbf{r}\right)} \cdot \overline{v_{j}\left(\mathbf{x}_{0}+\mathbf{r}\right)}+\sigma_{i j}\left(\mathbf{x}_{0}+\mathbf{r}\right) .
$$

We already know that $\overline{\mathbf{v}}$ is constant inside $\delta V$, so that

$$
\nabla_{\mathbf{r}} \cdot \mathbf{M}(\mathbf{r})=\nabla_{\mathbf{r}} \cdot \boldsymbol{\sigma}^{(\mathrm{R})}\left(\mathbf{x}_{0}+\mathbf{r}\right)=\nabla_{\mathbf{x}} \cdot \boldsymbol{\sigma}^{(\mathrm{R})}(\mathbf{x}),
$$

where $\mathbf{x}=\mathbf{x}_{0}+\mathbf{r}$. Therefore, by the averaged (NSE) (5.11), we get the relation

$$
\nabla_{\mathbf{r}} \cdot \mathbf{M}(\mathbf{r})=\mathbf{f}-\nabla_{\mathbf{x}} \bar{p}(\mathbf{x}) .
$$

(ii) — The incompressibility condition yields

$$
\nabla_{\mathbf{r}} \cdot \mathbf{N}(\mathbf{r})=0
$$

(iii) — A similar argmentation as in point (i) allows to write

$$
P_{i j}(\mathbf{r})=\overline{v_{i}\left(\mathbf{x}_{0}+\mathbf{r}\right)} \cdot \overline{v_{j}\left(\mathbf{x}_{0}\right)}+\overline{v_{i}^{\prime}\left(\mathbf{x}_{0}+\mathbf{r}\right) v_{j}^{\prime}\left(\mathbf{x}_{0}\right)} .
$$

As $\overline{\mathbf{v}}$ is constant in $\delta V$, we get $\nabla_{\mathbf{r}} \cdot\left[\overline{v_{i}\left(\mathbf{x}_{0}+\mathbf{r}\right)} \cdot \overline{v_{j}\left(\mathbf{x}_{0}\right)}\right]=0$. The difficult term to deal with is the second one in the r.h.s (6.21). A Taylor expansion yields

$$
v_{i}^{\prime}\left(\mathbf{x}_{0}+\mathbf{r}\right)=v_{i}^{\prime}\left(\mathbf{x}_{0}\right)+\nabla_{\mathbf{x}} v_{i}^{\prime}\left(\mathbf{x}_{0}\right) \cdot \mathbf{r}+\left(H\left(v_{i}^{\prime}\right)\left(\mathbf{x}_{0}\right) \mathbf{r}, \mathbf{r}\right)+r^{3} \eta_{i}(\mathbf{r}),
$$

where $\eta_{i}$ is some bounded function, $H\left(v_{i}^{\prime}\right)\left(\mathbf{x}_{0}\right)$ the Hessian matrix of $v_{i}^{\prime}$ at $\mathbf{x}_{0}$, which means

$$
\left(H\left(v_{i}^{\prime}\right)\left(\mathbf{x}_{0}\right) \mathbf{r}, \mathbf{r}\right)=\frac{\partial^{2} v_{i}^{\prime}}{\partial x_{p} \partial x_{q}}\left(\mathbf{x}_{0}\right) r_{p} r_{q} .
$$

On one hand, $\nabla_{\mathbf{r}} \cdot \overline{v_{i}^{\prime}\left(\mathbf{x}_{0}\right) v_{j}^{\prime}\left(\mathbf{x}_{0}\right)}=0$. On the other hand, a rather straightforward calculation yields

$$
\frac{\partial}{\partial r_{j}} \overline{\left(\frac{\partial v_{i}^{\prime}}{\partial x_{q}}\left(\mathbf{x}_{0}\right) r_{q} v_{j}^{\prime}\left(\mathbf{x}_{0}\right)\right)}=\overline{\frac{\partial v_{i}^{\prime}}{\partial x_{j}}\left(\mathbf{x}_{0}\right) v_{j}^{\prime}\left(\mathbf{x}_{0}\right)}=\frac{\partial}{\partial x_{j}}\left(\overline{v_{i}^{\prime}\left(\mathbf{x}_{0}\right) v_{j}\left(\mathbf{x}_{0}\right)}\right)=\frac{\partial \sigma_{i j}}{\partial x_{j}}\left(\mathbf{x}_{0}\right),
$$

where we have used the incompressibility condition combined with the Reynolds rule (5.6). Finally, let $\boldsymbol{\tau}^{(\mathrm{R})}$ denotes the tensor

$$
\boldsymbol{\tau}^{(\mathrm{R})}=\overline{\nabla_{\mathbf{x}} \mathbf{v}^{\prime} \otimes \mathbf{v}^{\prime}}=\left(\overline{\frac{\partial v_{i}^{\prime}}{\partial x_{k}} v_{j}^{\prime}}\right)_{1 \leq i j k \leq 3}=\left(\tau_{i j k}\right)_{1 \leq i j k \leq 3} .
$$

Using the incompressibility condition again and the Reynolds rule (5.6), we get

$$
\frac{\partial}{\partial r_{j}} \overline{\left(H\left(v_{i}^{\prime}\right)\left(\mathbf{x}_{0}\right) \mathbf{r}, \mathbf{r}\right) v_{j}^{\prime}\left(\mathbf{x}_{0}\right)}=\frac{\partial \tau_{i j k}}{\partial x_{j}} r_{k}
$$

Therefore, these calculations lead to

$$
\nabla_{\mathbf{r}} \cdot \mathbf{P}=\nabla_{\mathbf{x}} \cdot \boldsymbol{\sigma}^{(\mathrm{R})}\left(\mathbf{x}_{0}\right)+\nabla_{\mathbf{x}} \cdot \boldsymbol{\tau}^{(\mathrm{R})}\left(\mathbf{x}_{0}\right) \cdot \mathbf{r}+r^{2} \boldsymbol{\eta}(\mathbf{r}),
$$

which yields, by the averaged (NSE) (5.11),

$$
\nabla_{\mathbf{r}} \cdot \mathbf{P}=\mathbf{f}-\nabla_{\mathbf{x}} \bar{p}\left(\mathbf{x}_{0}\right)+\nabla_{\mathbf{x}} \cdot \boldsymbol{\tau}^{(\mathrm{R})}\left(\mathbf{x}_{0}\right) \cdot \mathbf{r}+r^{2} \boldsymbol{\eta}(\mathbf{r}) .
$$


(iv) - As $\mathbf{Q}$ does not depend on $\mathbf{r}$, we obviously have

$$
\nabla_{\mathbf{r}} \cdot \mathbf{Q}=0
$$

Step 3. By combining (6.15), (6.19) , (6.20), (6.27) and (6.28), we obtain the asymptotic expansion

$$
\nabla_{\mathbf{r}} \cdot B=-\nabla_{\mathbf{x}} \overline{q(\mathbf{r})}-\nabla_{\mathbf{x}} \cdot \boldsymbol{\tau}^{(\mathrm{R})}\left(\mathbf{x}_{0}\right) \cdot \mathbf{r}-r^{2} \boldsymbol{\eta}(\mathbf{r})
$$

by setting

$$
q(\mathbf{r})=q\left(\mathbf{x}_{0}+\mathbf{r}\right)-q\left(\mathbf{x}_{0}\right) .
$$

As we have assumed that the mean pressure gradient is constant inside $\delta V$, the firts term in the r.h.s of (6.29) vanishes, so that

$$
\nabla_{\mathbf{r}} \cdot B=-\nabla_{\mathbf{x}} \cdot \boldsymbol{\tau}^{(\mathrm{R})}\left(\mathbf{x}_{0}\right) \cdot \mathbf{r}-r^{2} \boldsymbol{\eta}(\mathbf{r}),
$$

that we combine with (6.11), which leads to

$$
\left[\left(3 \alpha_{d}-\alpha_{n}\right) \mathrm{Id}+\nabla_{\mathbf{x}} \cdot \boldsymbol{\tau}^{(\mathrm{R})}\left(\mathbf{x}_{0}\right)\right] \cdot \mathbf{r}+r^{2} \gamma(\mathbf{r})=0,
$$

for some bounded function $\gamma$. Since this equality holds regardless of $\mathbf{r}$, whose norm is small, we deduce

$$
\left(3 \alpha_{d}-\alpha_{n}\right) \operatorname{Id}+\nabla_{\mathbf{x}} \cdot \boldsymbol{\tau}^{(\mathrm{R})}\left(\mathbf{x}_{0}\right)=0 .
$$

We observe that the diagonal coefficients of the matrix $\nabla_{\mathbf{x}} \cdot \boldsymbol{\tau}^{(\mathrm{R})}\left(\mathrm{x}_{0}\right)$ satisfy

$$
\frac{\partial \tau_{i j i}}{\partial x_{j}}=\frac{\partial}{\partial x_{j}}\left(\overline{\frac{\partial v_{i}^{\prime}}{\partial x_{i}} v_{j}^{\prime}}\right)=0,
$$

because of the incompressibilty condition. Consequently, the following holds:

$$
\begin{aligned}
& 3 \alpha_{d}=\alpha_{n}, \\
& \nabla_{\mathbf{x}} \cdot \boldsymbol{\tau}^{(\mathrm{R})}\left(\mathbf{x}_{0}\right)=0,
\end{aligned}
$$

in particular

$$
B_{n}(r)=3 B_{d}(r)+o\left(r^{3}\right),
$$

hence formula (6.8) by setting $E(r)=-2 B_{d}(r)$.

Remark 6.1. The assumption that the gradient of the mean pressure is constant is not too restrictive. Experiments suggest that this is indeed verified by flows in pipes or in boundary layers. However, the general case remains an open problem.

Remark 6.2. In [21] the general two order correlation tensor is considered as a bilinear form, which directly provides the formula

$$
B(\mathbf{r})=E(r) \frac{\mathbf{r} \otimes \mathbf{r}}{r^{2}}-\frac{3}{2} E(r) \mathrm{I}_{3},
$$

without using the NSE. We do not know how to prove this bilinearity property. 


\subsection{Derivation of the law of the $2 / 3$}

We prove in this section the law of the $2 / 3$, specified by (6.51) below, under assumption 6.1. The previous section states that,

$$
B(\mathbf{r}) \sim E(r) \frac{\mathbf{r} \otimes \mathbf{r}}{r^{2}}-\frac{3}{2} E(r) \mathrm{I}_{3},
$$

and near 0

$$
E(r) \approx r^{2}
$$

The function $E(r)$ is obviously defined in $[0, \ell]$. The question is the behavior of $E(r)$ when $r$ differs from 0 . According to Kolmogorov [21], we assume that $E$ is entirely driven in $\delta V$ by the kinematic viscosity $\nu$ and the mean dissipation at $\mathbf{x}_{0}$, specified by

$$
\mathscr{E}=\overline{2 \nu\left|D \mathbf{v}\left(\mathbf{x}_{0}\right)\right|^{2}} .
$$

It is easily checked that $\nu$ and $\mathscr{E}$ are dimensionally independant. We deduce from these quantities the length-time basis $b_{0}=\left(\lambda_{0}, \tau_{0}\right)$, where

$$
\lambda_{0}=\nu^{\frac{3}{4}} \mathscr{E}-\frac{1}{4}, \quad \tau_{0}=\nu^{\frac{1}{2}} \mathscr{E}-\frac{1}{2} .
$$

The lenght scale $\lambda_{0}$ is known as the "Kolmogorov scale". Following Definition 3.2 and by the table in section 3.1 , we get

$$
\forall r^{\prime} \in\left[0, \frac{\ell}{\lambda_{0}}\left[, \quad E\left(\lambda_{0} r^{\prime}\right)=(\nu \mathscr{E})^{\frac{1}{2}} E_{b_{0}}\left(r^{\prime}\right) .\right.\right.
$$

We first assume that

$$
\lambda_{0}<<\ell,
$$

so that we can consider as a first approximation for the simplicity,

$$
\left[0, \frac{\ell}{\lambda_{0}}\left[\sim \mathbb{R}_{+}\right.\right.
$$

The main Kolmogorov assumption can be translated as follows: there is a range $\left[r_{1}, r_{2}\right]$, satisfying

$$
\lambda_{0}<<r_{1}<<r_{2}<<\ell,
$$

and such that in the range $\left[r_{1}, r_{2}\right], E$ is uniquely determined by $\mathscr{E}$. This last sentence can lead to confusion, and the concept needs to be made more specific and rigorous by a definition similar to Definition 3.3, based on a similarity statement. Therefore we shall assume the following:

Assumption 6.1. For all lenght-times bases $b_{1}=\left(\lambda_{1}, \tau_{1}\right)$ and $b_{2}=\left(\lambda_{2}, \tau_{2}\right)$,

$$
\mathscr{E}_{b_{1}}=\mathscr{E}_{b_{2}} \quad \Rightarrow \quad \forall r^{\prime} \in\left[\frac{r_{1}}{\lambda_{1}}, \frac{r_{2}}{\lambda_{1}}\right] \cap\left[\frac{r_{1}}{\lambda_{2}}, \frac{r_{2}}{\lambda_{2}}\right], \quad E_{b_{1}}\left(r^{\prime}\right)=E_{b_{2}}\left(r^{\prime}\right) .
$$

In the following, we set

$$
r_{1,0}^{\prime}=\frac{r_{1}}{\lambda_{0}}, \quad r_{2,0}^{\prime}=\frac{r_{2}}{\lambda_{0}}
$$


Proposition 6.1. If assumption 6.1 holds, then there exists a constant $C$ such that

$$
\forall r^{\prime} \in\left[r_{1,0}^{\prime}, r_{2,0}^{\prime}\right], \quad E_{b_{0}}\left(r^{\prime}\right)=C\left(r^{\prime}\right)^{\frac{2}{3}} .
$$

Proof. The proof is based on the determination of a functional equation satisafied by $E_{b_{0}}$. Let $\alpha>0$, and $b^{(\alpha)}=\left(\alpha^{3} \lambda_{0}, \alpha^{2} \tau_{0}\right)$. This choice is motivated by the equality

$$
\forall \alpha>0, \quad \mathscr{E}_{b^{(\alpha)}}=\mathscr{E}_{b_{0}},
$$

which is easily checked. Therefore,

$$
\forall r^{\prime} \in I_{\alpha}=\left[r_{1,0}^{\prime}, r_{2,0}^{\prime}\right] \cap\left[\frac{r_{1,0}^{\prime}}{\alpha^{3}}, \frac{r_{2,0}^{\prime}}{\alpha^{3}}\right], \quad E_{b^{(\alpha)}}\left(r^{\prime}\right)=E_{b_{0}}\left(r^{\prime}\right),
$$

which is consistant so far

$$
\frac{r_{1}}{r_{2}}<\alpha<\frac{r_{2}}{r_{1}} .
$$

According to (6.42), statement (6.46) makes sense for a large range of $\alpha \in \mathbb{R}_{+}^{\star}$. By Definition 3.2, we get

$$
E\left(\lambda_{0} \alpha^{3} r^{\prime}\right)=\alpha^{2}(\nu \mathscr{E})^{\frac{1}{2}} E_{b^{(\alpha)}}\left(r^{\prime}\right)
$$

hence, $\forall r^{\prime} \in I_{\alpha}$,

$$
E_{b^{(\alpha)}}\left(r^{\prime}\right)=\frac{1}{\alpha^{2}(\nu \mathscr{E})^{\frac{1}{2}}} E\left(\lambda_{0} \alpha^{3} r^{\prime}\right)=E_{b_{0}}\left(r^{\prime}\right)
$$

where we have used (6.46). We combine (6.40) with (6.48), which yields

$$
E_{b_{0}}\left(r^{\prime}\right)=\frac{1}{\alpha^{2}} E_{b_{0}}\left(\alpha^{3} r^{\prime}\right)
$$

We deduce from equation (6.49) by standard calculations

$$
\forall r^{\prime} \in\left[r_{1,0}^{\prime}, r_{2,0}^{\prime}\right], \quad E_{b_{0}}\left(r^{\prime}\right)=\left(\frac{E_{b_{0}}\left(r_{1}^{\prime}\right)}{\left(r_{1}^{\prime}\right)^{\frac{2}{3}}}\right)\left(r^{\prime}\right)^{\frac{2}{3}},
$$

which concludes the proof.

In conclusion, combining (6.39), (6.40) and (6.50) by writting $r^{\prime}=r / \lambda_{0}$, we get

$$
\forall r \in\left[r_{1}, r_{2}\right], \quad E(r)=C(\mathscr{E} r)^{\frac{2}{3}},
$$

which is indeed the law of the $2 / 3$ as initially found in [21, 29].

\section{References}

[1] J. Boussinesq. Theorie de l'écoulement tourbillant. Mém. prés par div. savants á la Acad. Sci., 23:46-50, 1877.

[2] L. Caffarelli, R. Kohn, and L. Nirenberg. Partial regularity of suitable weak solutions of the Navier-Stokes equations. Comm. Pure Appl. Math., 35(6):771-831, 1982.

[3] M. Cannone. Harmonic analysis tools for solving the incompressible Navier-Stokes equations. In Handbook of mathematical fluid dynamics. Vol. III, pages 161-244. North-Holland, Amsterdam, 2004. 
[4] M. Cannone and Y. Meyer. Littlewood-Paley decomposition and Navier-Stokes equations. Methods Appl. Anal., 2(3):307-319, 1995.

[5] T. Chacòn-Rebollo and R. Lewandowski. Mathematical and numerical foundations of turbulence models and applications. Birkäuser, Springer, New-York, 2014.

[6] J-Y. Chemin. About weak-strong uniqueness for the 3D incompressible Navier-Stokes system. Comm. Pure Appl. Math., 64(12):1587-1598, 2011.

[7] H.-J. Choe and J.-L. Lewis. On the singular set in the Navier-Stokes equations. $J$. Funct. Anal., 175(2):348-369, 2000.

[8] P. Constantin, C.R. Doering, and E.S. Titi. Rigorous estimates of small scales in turbulent flows. J. Math. Phys., 37(12):6152-6156, 1996.

[9] P. Constantin and C. Foias. Navier-Stokes equations. Chicago Lectures in Mathematics. University of Chicago Press, Chicago, IL, 1988.

[10] C.R. Doering and C. Foias. Energy dissipation in body-forced turbulence. J. Fluid Mech., 467:289-306, 2002.

[11] C.R. Doering and J.D. Gibbon. Applied analysis of the Navier-Stokes equations. Cambridge Texts in Applied Mathematics. Cambridge University Press, Cambridge, 1995.

[12] C.R. Doering and E.S. Titi. Exponential decay rate of the power spectrum for solutions of the Navier-Stokes equations. Phys. Fluids, 7(6):1384-1390, 1995.

[13] C.L. Fefferman. Existence and smoothness of the Navier-Stokes equation. In The millennium prize problems, pages 57-67. Clay Math. Inst., Cambridge, MA, 2006.

[14] E. Feireisl. Dynamics of viscous incompressible fluids. Oxford University Press, 2004.

[15] C. Foias, M. S. Jolly, O. P. Manley, R. Rosa, and R. Temam. Kolmogorov theory via finite-time averages. Phys. D, 212(3-4):245-270, 2005.

[16] C. Foias, O. Manley, R. Rosa, and R. Temam. Navier-Stokes equations and turbulence, volume 83 of Encyclopedia of Mathematics and its Applications. Cambridge University Press, Cambridge, 2001.

[17] H. Fujita and T. Kato. On the Navier-Stokes initial value problem. Arch. Rational Mech. Anal., 16:269-315, 1964.

[18] J.D. Gibbon and C.R. Doering. Intermittency and regularity issues in 3D NavierStokes turbulence. Arch. Ration. Mech. Anal., 177(1):115-150, 2005.

[19] E. Hopf. Über die Anfangswertaufgabe für die hydrodynamischen Grundgleichungen. Math. Nachr., 4:213-231, 1951.

[20] H. Koch and D. Tataru. Well-posedness for the Navier-Stokes equations. Adv. Math., 157(1):22-35, 2001.

[21] A.N. Kolmogorov. The local structure of turbulence in incompressible viscous fluids for very large Reynolds number. Dokl. Akad. Nauk SSR, 30:9-13, 1941. 
[22] J. Leray. Sur le mouvement d'un liquide visqueux emplissant l'espace. Acta Math., 63(1):193-248, 1934.

[23] F. Lin. A new proof of the Caffarelli-Kohn-Nirenberg theorem. Comm. Pure Appl. Math., 51(3):241-257, 1998.

[24] J.-L. Lions. Quelques méthodes de résolution des problèmes aux limites non linéaires. Dunod; Gauthier-Villars, Paris, 1969.

[25] P-L. Lions. Mathematical topics in fluid mechanics. Vol. 1, volume 3 of Oxford Lecture Series in Mathematics and its Applications. The Clarendon Press, Oxford University Press, New York, 1996. Incompressible models, Oxford Science Publications.

[26] L. Prandtl. über die ausgebildeten turbulenz. Zeitschrift für angewandte Mathematik und Mechanik, 5:136-139, 1925.

[27] O. Reynolds. An experimental investigation of the circumstances which determine whether the motion of water shall be direct or sinuous, and of the law of resistance in parallel channels. Philosophical Transactions of the Royal Society, 174:935-982, 1883.

[28] V. Scheffer. Hausdorff measure and the Navier-Stokes equations. Comm. Math. Phys., 55(2):97-112, 1977.

[29] G.I. Taylor. Statistical theory of turbulence, part i-iv. Proc. Roy. Soc. A, 151:421-478, 1935.

[30] R. Temam. Navier-Stokes equations. AMS Chelsea Publishing, Providence, RI, 2001. Theory and numerical analysis, Reprint of the 1984 edition. 